\title{
Molecular analysis of human endometrium: short-term tibolone signaling differs significantly from estrogen and estrogen + progestagen signaling
}

\author{
P. Hanifi-Moghaddam • B. Boers-Sijmons • \\ A. H. A. Klaassens - F. H. van Wijk • \\ M. A. den Bakker • M. C. Ott • G. L. Shipley • \\ H. A. M. Verheul • H. J. Kloosterboer • C. W. Burger • \\ L. J. Blok
}

Received: 31 August 2006/Revised: 13 November 2006 / Accepted: 23 November 2006 / Published online: 17 January 2007

(C) Springer-Verlag 2007

\begin{abstract}
Tibolone, a tissue-selective compound with a combination of estrogenic, progestagenic, and androgenic properties, is used as an alternative for estrogen or estrogen plus progesterone hormone therapy for the treatment of symptoms associated with menopause and osteoporosis. The current study compares the endometrial gene expression profiles after short-term (21 days) treatment with tibolone to the profiles after treatment with estradiol-only
\end{abstract}

P. Hanifi-Moghaddam • L. J. Blok $(\bowtie)$

Department of Reproduction and Development,

Erasmus University Medical Center,

P.O. Box 1738, 3000 DR Rotterdam, The Netherlands

e-mail: 1.blok@erasmusmc.nl

B. Boers-Sijmons · A. H. A. Klaassens · F. H. van Wijk •

C. W. Burger $\cdot$ L. J. Blok

Department of Obstetrics and Gynecology,

Erasmus University Medical Center,

Rotterdam, The Netherlands

M. A. den Bakker

Department of Pathology, Erasmus University Medical Center,

Rotterdam, The Netherlands

\section{C. Ott}

OmniViz, Inc.,

Maynard, MA, USA

G. L. Shipley

Department of Integrative Biology and Pharmacology,

University of Texas Houston Health Science Center,

Houston, TX, USA

H. A. M. Verheul · H. J. Kloosterboer

N.V. Organon,

Oss, The Netherlands

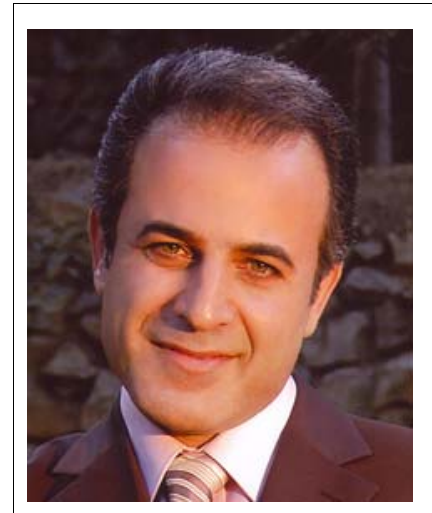

Payman Hanifi Moghaddam received his Ph.D. in Immunogenetics from Leiden University Medical Centre, The Netherlands. He is presently a Senior Research Scientist at the Department of Molecular Design and Bioinformatics at Organon N.V., Oss, The Netherlands. His current research interests include system biology and complex diseases.

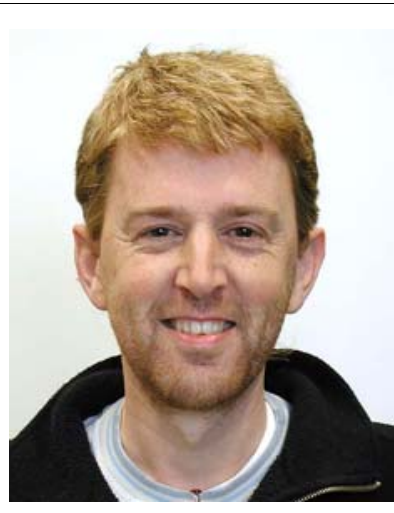

LEEN J BLOK

received his Ph.D. in Biochemistry from the Erasmus University Rotterdam, The Netherlands. He is presently an Assistant Professor at the Department of Obstetrics and Gynaecology at the Erasmus University Medical Center Rotterdam, The Netherlands. His current work involves studies into normal and aberrant hormone signaling in the female urogenital tract.
$\left(E_{2}\right)$ and $E_{2}+$ medroxyprogesterone acetate $\left(E_{2}+M P A\right)$ in healthy postmenopausal women undergoing hysterectomy for endometrial prolapse. The impact of $\mathrm{E}_{2}$ treatment on endometrial gene expression (799 genes) was much higher than the effect of tibolone (173 genes) or $E_{2}+$ MPA treatment (174 genes). Furthermore, endometrial gene expression profiles after tibolone treatment show a weak similarity to the profiles after $\mathrm{E}_{2}$ treatment (overlap 72 
genes) and even less profile similarity to $E_{2}+$ MPA treatment (overlap 17 genes). Interestingly, 95 tibolonespecific genes were identified. Translation of profile similarity into biological processes and pathways showed that ER-mediated downstream processes, such as cell cycle and cell proliferation, are not affected by E2 + MPA, slightly by tibolone, but are significantly affected by $E_{2}$. In conclusion, tibolone treatment results in a tibolone-specific gene expression profile in the human endometrium, which shares only limited resemblance to $E_{2}$ and even less resemblance to $\mathrm{E} 2+\mathrm{MPA}$ induced profiles.

Keywords Tibolone $\cdot$ Endometrium $\cdot$ Hormone replacement therapy · Estrogen · Progestagen · Gene expression profiling

\section{Introduction}

Unopposed estrogen treatment increases the risk for endometrial hyperplasia and subsequent carcinoma. For example in the HOPE study (Women's Health, Osteoporosis, Progestin, Estrogen study $[1,2])$ after 2 years of treatment with conjugated equine estrogens (CEE; $0.625 \mathrm{mg} /$ day), the rate of hyperplasia went up to $27.7 \%$. When the treatment was combined with medroxyprogesterone acetate (MPA; $2.5 \mathrm{mg}$ ), incidence of endometrial hyperplasia decreased to the baseline incidence. Thus, the addition of progestagens is necessary for endometrial safety. Progestagen addition, however, also causes negative side effects like withdrawal bleeding and an increased breast cancer incidence that was recently documented in the Women's Health Initiative [3] and Million Women Study [4].

Tibolone is a synthetic steroid with a unique clinical profile within the currently available postmenopausal therapeutic options. Tibolone shows a tissue-selective mode of action; in the vagina, brain, and bone, tibolone acts as an estrogen, whereas no or slight estrogenic stimulation has been found in breast and endometrium [5-7]. The tissueselective mode of action of tibolone and its metabolites is due to the interplay between several mechanisms including tissue-selective metabolism, enzymatic inactivation or activation, and differential receptor binding.

During passage through the gastrointestinal tract and the liver, tibolone is converted into three metabolites: two $\mathrm{OH}-$ metabolites, $3 \alpha-\mathrm{OH}$-tibolone and $3 \beta-\mathrm{OH}$-tibolone (exerting estrogenic effects and in vivo present in large amounts) [8], and the $\Delta^{4}$-isomer (exerting progestagenic and androgenic effects, and in vivo in plasma only present for a relatively short period of time) $[5,9]$. Besides the metabolism in the liver and intestine, metabolism of tibolone also takes place inside target tissues. For example, the lack of stimulation of the human endometrium after long-term tibolone treatment may partly be explained by the dominant presence of the
$\Delta^{4}$-isomer of tibolone. Evidence in support of this tissueselective metabolism has been found in several in vitro test systems. Tang et al. [10] have demonstrated that tibolone can locally be converted in endometrial explants to the $\Delta^{4}$ isomer, and conversion of the 3-OH-metabolites back to tibolone, and subsequently to the $\Delta^{4}$-isomer, is another possible mechanism that contributes to the local formation of $\Delta^{4}$-isomer [11]. Furthermore, our own work showed that in an endometrial cancer cell line, the presence of bioactive $\Delta^{4}$-isomer could indeed effectively counteract the estrogenic activity of tibolone [12, 13] resulting in a clear predominant progestagenic effect.

In vivo, long-term tibolone use has been shown not to stimulate the human endometrium, although in clinical practice, especially at the beginning of a treatment, increased episodes of spotting and bleeding are observed. In a clinical study, for the first time, we investigated the short-term (21 days) effects of tibolone on endometrial histology and gene expression. Previously, we published the histological data [14] and biochemical findings [15], and now we present the molecular changes observed by analyzing gene expression profiles. The effects of shortterm (21 days) treatment with tibolone were compared to the effects of estradiol alone $\left(\mathrm{E}_{2}\right)$ and $\mathrm{E} 2+\mathrm{MPA}$.

\section{Materials and methods}

\section{Participants}

This study was designed as a controlled clinical trial. The trial was registered with National Institutes of Health, registry number NCT00294463, found at http://www.clinicaltrials. gov, under the name of Effects of Tibolone Treatment on the Endometrium. Patients who visited our clinics to undergo vaginal hysterectomy for treatment of prolapse were eligible to participate in this study. A description of the inclusion and exclusion criteria and the histological findings of the current experiments were documented earlier [14].

The trial was performed in the period before the scheduled surgery. After informed consent, the patients were sequentially assigned to one of the following treatment groups: control group (no hormonal treatment); tibolone group (2.5 mg tibolone (Livial, N.V. Organon, Oss, The Netherlands) administered orally every day, starting 21 days prior to surgery); $\mathrm{E}_{2}$ group ( $2 \mathrm{mg}$ of estradiol administered orally every day, starting 21 days before surgery); E2 + MPA group ( $2 \mathrm{mg}$ estradiol $+5 \mathrm{mg}$ MPA administered orally every day, starting 21 days before surgery). The investigators were kept blinded to the patient treatments during sex hormone-binding globulin (SHBG) measurements (Fig. 1), the cluster and correlation (Fig. 2) analyses, and during validation reverse transcription poly- 


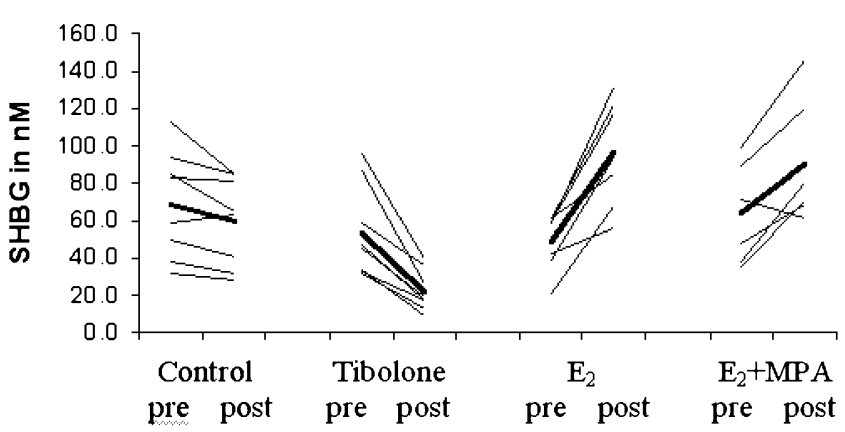

Fig. 1 SHBG measurements before and after treatment. Sera were collected for each patient just before the start of treatment (pre) and 21 days later just before hysterectomy (post). The bold lines represent the averages of the measured values

merase chain reaction (RT-PCR; Fig. 4). Investigations were conducted in accordance with the guidelines in The Declaration of Helsinki, and the local ethics committees of the participating hospitals approved the study protocol.

Measurement of sex hormone-binding globulin

Sera were collected before the start of treatment and at surgery, processed and stored at $-20^{\circ} \mathrm{C}$ until measurement. SHBG was measured in sera of the different patient groups by ABL (Assen, The Netherlands) with a commercially available and validated AutoDelphia immunoassay and using the automatic Delfia system 1235 (Wallac Oy, Turku, Finland). The accuracy ranged from 101.1 to $110.9 \%$ with a coefficient of variation expressed as percentage of the mean of $1.5-1.8 \%$. By measuring the SHBG values we were able to verify compliance of the patients to the treatments.

Isolation of pure endometrial tissue and subsequent RNA isolation

Immediately after hysterectomy (within $10 \mathrm{~min}$ ), part of the uterus was dissected and snap-frozen for RNA isolation. Frozen tissue samples were embedded and 5- $\mu \mathrm{m}$ sections were collected in batches of ten. Each first and last section in such a series was stained and evaluated under the microscope for the percentage of endometrium and myometrium. Only those batches with $100 \%$ pure endometrial tissue were selected for RNA isolation. Tissues were homogenized by sonication and RNA was isolated using Trizol (Invitrogen, Life Technologies, Philadelphia, PA, USA). The concentration, quality, and purity of RNA were assessed with the use of the RNA 6000 Nano assay on the Agilent 2100 Bioanalyzer (Agilent, Palo Alto, CA, USA). Only those RNA samples that showed no sign of degradation or contamination by DNA were used for gene expression profiling.

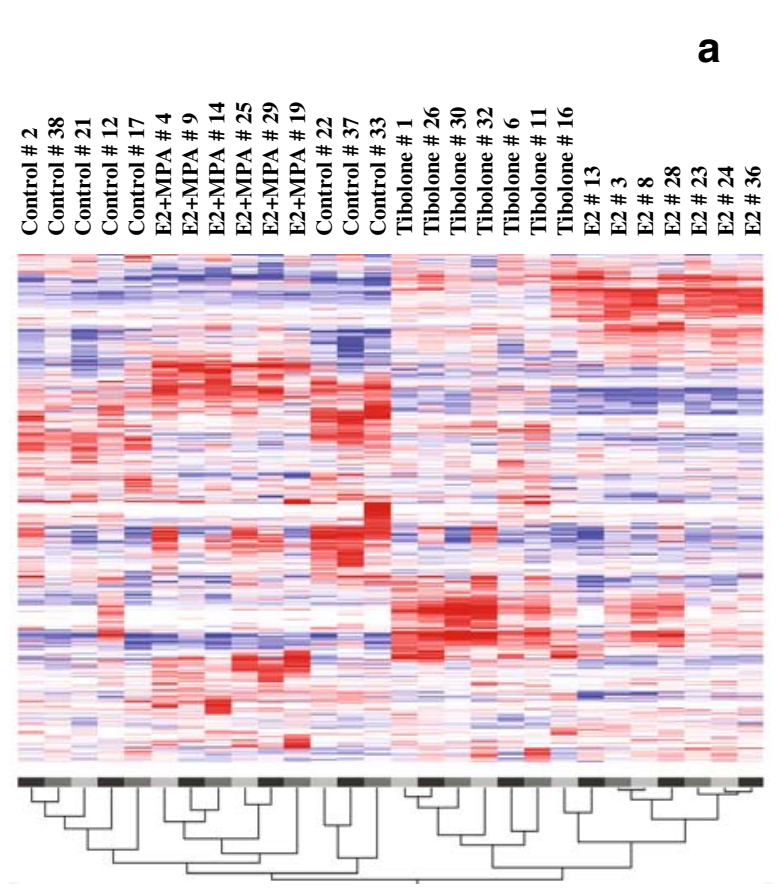

\section{b}

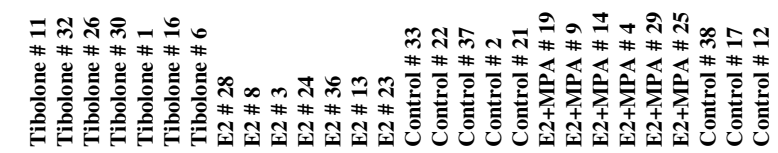

Tibolone \# 11

Tibolone \# 32

Tibolone \# 26

Tibolone \# 30

Tibolone \# 1

Tibolone \# 16

Tibolone \# 6

E2 \# 28

E2 \# 8

E2 \# 3

E2 \# 24

E2 \# 36

E2 \# 13

E2 \# 23

Control \# 33

Control \# 22

Control \# 37

Control \# 2

Control \# 21

E2+MPA \# 19

E2+MPA \# 9

E2+MPA \# 14

E2+MPA \# 4

E2+MPA \# 29

E2+MPA \# 25

Control \# 38

Control \# 17

Control \# 12

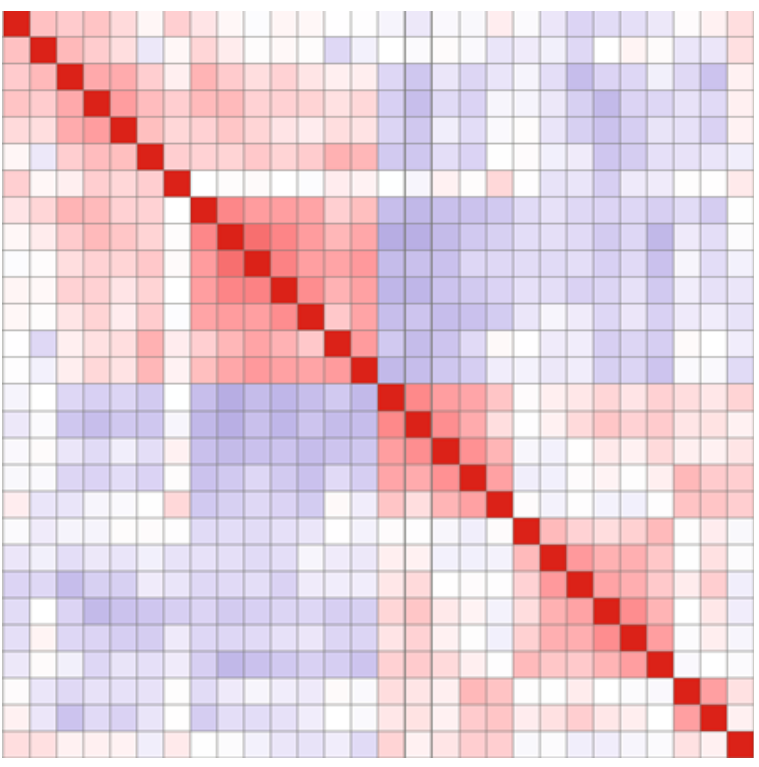

Fig. 2 Cluster and correlation analyses of endometrial gene expression profiles obtained after short-term (21 days) treatment with $E_{2}, E_{2}+M P A$, or tibolone. Using statistical analysis, it was established that 3,293 probe sets (representing 2,312 known and 466 unknown genes) deviated at least threefold, in at least one patient sample, from the geometrical means of all treated samples. These genes were used for the cluster and correlation analyses. a At the

bottom of the figure, relatedness is indicated. Red indicates genes with a higher expression relative to the geometrical means, while blue indicates genes with a lower expression relative to the geometrical means. The numbers (\#) behind the treatments indicate the patient numbers. b The correlation values are between 1 (dark red, diagonal line) and -1 (dark blue, not represented here). The white boxes represent no correlation between profiles 
Gene expression profiling

Gene expression profiles of samples were generated with the use of Affymetrix U133plus2 GeneChips containing 54,614 probe sets, representing approximately 47,000 transcripts (Affymetrix, Santa Clara, CA, USA). One microgram of total RNA was used to prepare antisense biotinylated RNA according to the Affymetrix protocol for gene chip experiments (http://www.affymetrix.com). The level and quality of cRNA was measured on the Agilent 2100 Bioanalyzer (Agilent). If a cRNA sample showed any abnormal pattern, this sample was not used for further analysis and new cRNA was generated. The cRNA was fragmented with the GeneChip Sample Cleanup Module (Affymetrix). Hybridization, staining, washing, and scanning procedures were carried out as described in the GeneChip Expression Analysis technical manual (Affymetrix). All GeneChips were visually inspected for irregularities. All array data is freely accessible from the Erasmus University Medical Center database (ENDO_GROUP PDATA at http://www.gatcplatform.nl/).

Data analyses

\section{Similarity and correlation between expression profiles}

Data normalization was done according to the quantile method [16]. Subsequently, all intensity values below 30 were set at 30 , since our methods reliably identify samples with an average intensity value of 30 or more but do not reliably discriminates values between 0 and 30 . For each probe set, the geometrical mean of the hybridization intensities of all samples from the subjects was calculated. The level of expression of each gene in every sample was determined relative to this geometric mean and logarithmically transformed (on a base 2 scale) to assign equal weight to gene expression levels with similar relative distances to the geometrical mean. Deviation from the geometrical mean reflects differential gene expression. Genes whose level of expression differed at least threefold, in at least one sample from the geometrical means of all treated samples (reflecting up- or downregulation), were selected for further analysis.

The similarity and correlation between expression profiles of different treatment groups was investigated with the use of cluster analysis and the Pearson's correlation and Visualization tool of Omniviz (Ominviz, Maynard, MA, USA; version 3.8).

\section{Identification of genes with significant differential expression}

We used significance analysis of microarrays (SAM) [17], implemented in Omniviz to determine genes with signifi- cantly differential expression between the treatment groups. SAM is a statistical method for identifying differentially expressed genes while controlling the overall false discovery rate (FDR). FDR is the percentage of genes identified by chance. We have chosen the settings for this analysis in such a way that the total number of falsely identified differential expressed genes was less than 1 (this means that the $p$ value for a certain SAM analysis is smaller than $1 /$ number of calculated differentially expressed genes).

\section{Functional and biological classification of genes}

The classification of genes into biological processes and functional analyses was done using DAVID (http:// david.abcc.ncifcrf.gov), Panther (http://www.panther. appliedbiosystems.com), and Ingenuity Pathway software (http://www.ingenuity.com). In order to identify biological processes with significantly enriched or depleted gene numbers, the distribution of genes in each hormoneregulated gene list for a particular biological process was compared to that in a reference gene list. Since an inappropriate reference list would lead to wrongful identification of regulated biological processes, we used two reference lists: the Affymetrix 133Uplus2 gene list and the NCBI human gene list $(n=23,481)$. The enrichment or depletion of genes, compared to the NCBI reference list, in a certain biological process indicates significant hormonal regulation $\left(p<10^{-4}\right)$. The reason for using different classification software and databases was to verify consistency of classification and to enhance reliability of the results.

\section{Real-time quantitative PCR verifications}

Validation of microarray expression data was accomplished by real-time quantitative PCR (RT-qPCR) on ten selected genes: CCND1 (cyclinD1),ER $\alpha$ (estrogen receptor $\alpha$ ), ER $\beta$ (estrogen receptor $\beta$ ), TFF3 (Trefoil factor 3), IGFBP3 (insulin-like growth factor binding protein 3), IGFI (insulin-like growth factor 1), VEGF (vascular endothelial growth factor), $P R$ (progesterone receptor), CSPG2 (versican), HSD17B2 (hydroxysteroid [17] dehydrogenase 2). RT-qPCR was done as previously described [18]. The sequences of primers and probes used in this study are available upon request.

\section{Results}

Participants

In total, 30 out of 33 eligible postmenopausal patients participated in this study. The $\mathrm{E}_{2}$ group originally included eight subjects, but one patient was excluded on the basis of 
a double layer endometrial thickness preceding $4 \mathrm{~mm}$. The E2 + MPA group originally also included eight subjects, but one patient was excluded on the basis of an abnormal cervical Pap smear test, while another patient had to be excluded because her surgery was rescheduled. Patients in the different groups were comparable with respect to age, number of menstrual years, years after menopause, endometrial thickness, and body mass index at screening [14].

Different steroid hormone treatments have been reported in the literature to result in specific changes in the SHBG concentrations: estrogen treatment will result in an increase in the SHBG levels while tibolone's androgenic actions in the liver will result in a reduction of the SHBG levels [19]. Therefore, SHBG levels were measured in serum because regular use of medication will be reflected in the SHBG levels. In Fig. 1, it is shown that $\mathrm{E}_{2}$ and $\mathrm{E} 2+\mathrm{MPA}$ treatments indeed resulted in a significant increase in serum SHBG levels in all, except one, subjects, while tibolone treatment resulted in a significant decrease in SHBG levels in all treated subjects.

\section{Similarity and correlation between expression profiles}

Cluster and correlation analysis allows us to visually assess the similarity between and within groups. The unsupervised cluster analysis (Fig. 2a) show that endometrial profiles of control and E2 + MPA treated patients cluster together. The fact that control samples cluster in two subgroups indicates a high intra-individual variation. The endometrial profiles of tibolone- and $E_{2}$-treated patients form the second main cluster. In this cluster, most endometrial profiles from tibolone- and $\mathrm{E}_{2}$-treated patients separate in two subclusters, indicating a higher profile correlation within both treatment groups than between treatment groups.

In order to measure the magnitude of profile similarity between treated individual patients and thus further differentiate our analysis, a correlation analysis was performed (Fig. 2b) and the average correlation data (calculated from the individual correlations depicted in Fig. 2b) per group are summarized in Table 1 . When we only consider those average correlations which deviate from zero, it is observed
Table 2 Number of significantly differentially regulated genes in each treatment group

\begin{tabular}{|c|c|c|c|c|c|c|c|}
\hline & & \multicolumn{2}{|c|}{$\mathrm{E}_{2}+\mathrm{MPA}$} & \multicolumn{2}{|l|}{$\mathrm{E}_{2}$} & \multicolumn{2}{|c|}{ Tibolone } \\
\hline & & Up & Down & Up & Down & $\mathrm{Up}$ & Down \\
\hline & & 82 & 93 & 535 & 265 & 146 & 28 \\
\hline \multirow[t]{2}{*}{$\mathrm{E}_{2}+\mathrm{MPA}$} & $\mathrm{Up}$ & 82 & & 15 & 1 & 8 & 0 \\
\hline & Down & 93 & & 0 & 27 & 0 & 9 \\
\hline \multirow[t]{2}{*}{$E_{2}$} & Up & 535 & & & & 55 & 0 \\
\hline & Down & 265 & & & & 0 & 17 \\
\hline \multirow{2}{*}{$\begin{array}{l}\text { Treatment- } \\
\text { specific }\end{array}$} & $\mathrm{Up}$ & 63 & & 470 & & 88 & \\
\hline & Down & & 63 & & 226 & & 8 \\
\hline
\end{tabular}

The number of significantly differentially regulated genes in each treatment group was identified relative to controls using SAM analysis. The overlap in gene lists between different treatment groups is shown. The numbers in italics indicate genes that are uniquely regulated by a specific treatment. Furthermore, five genes were upregulated by E2, E2 + MPA, and tibolone, and six genes were downregulated by all three treatments.

that the endometrial profiles from $E_{2}$-treated patients are negatively correlated to the endometrial profiles from control $(-0.26 \pm 0.13)$ and E2 + MPA $(-0.25 \pm 0.07)$ treated patients and slightly positively correlated to the endometrial profiles from tibolone $(+0.18 \pm 0.17)$ treated patients. Furthermore, the endometrial profiles from tibolone-treated patients show a small negative correlation to the profiles from E2 + MPA $(-0.17 \pm 0.13)$ treated patients. These correlation values support and further refine the cluster outcome. Furthermore, high intra-individual variation was observed within all groups.

\section{Identification of genes with significant differential expression}

To take the analysis one step further, SAM analysis was used to identify genes showing significant differential expression in each treatment group relative to control and relative to each other. The result is summarized in Table 2 . Relative to the control group, 799 genes are regulated in the endometria of $E_{2}$-treated patients, whereas 173 genes are significantly regulated in endometria from tibolone-treated

Table 1 Correlations between the different treatments

\begin{tabular}{|c|c|c|c|c|c|c|c|c|c|c|c|c|}
\hline $\begin{array}{l}\text { Average of } \\
\text { correlations }\end{array}$ & Control & Max & Min & $\mathrm{E} 2+\mathrm{MPA}$ & Max & Min & E2 & Max & Min & Tibolone & Max & Min \\
\hline Control & $\begin{array}{l}+0.34 \\
( \pm 0.16)\end{array}$ & 0.63 & 0.04 & $+0.04( \pm 0.14)$ & 0.38 & -0.17 & $-0.26( \pm 0.13)$ & 0.05 & -0.44 & $-0.12( \pm 0.18)$ & 0.27 & -0.39 \\
\hline $\mathrm{E} 2+\mathrm{MPA}$ & & & & $+0.46( \pm 0.12)$ & 0.63 & 0.23 & $-0.25( \pm 0.07)$ & -0.01 & -0.40 & $-0.17( \pm 0.13)$ & 0.08 & -0.40 \\
\hline E2 & & & & & & & $+0.55( \pm 0.10)$ & 0.77 & 0.32 & $+0.18( \pm 0.17)$ & 0.53 & -0.27 \\
\hline Tibolone & & & & & & & & & & $+0.36( \pm 0.28)$ & 0.61 & -0.09 \\
\hline
\end{tabular}

Average correlations calculated from the individual correlations depicted in Fig. 2a. Max represents the highest calculated individual correlation coefficient; min represents the lowest calculated correlation coefficient. All average correlations were calculated \pm SD. 
a

$\mathrm{E}_{2}$ regulated cell cycle genes

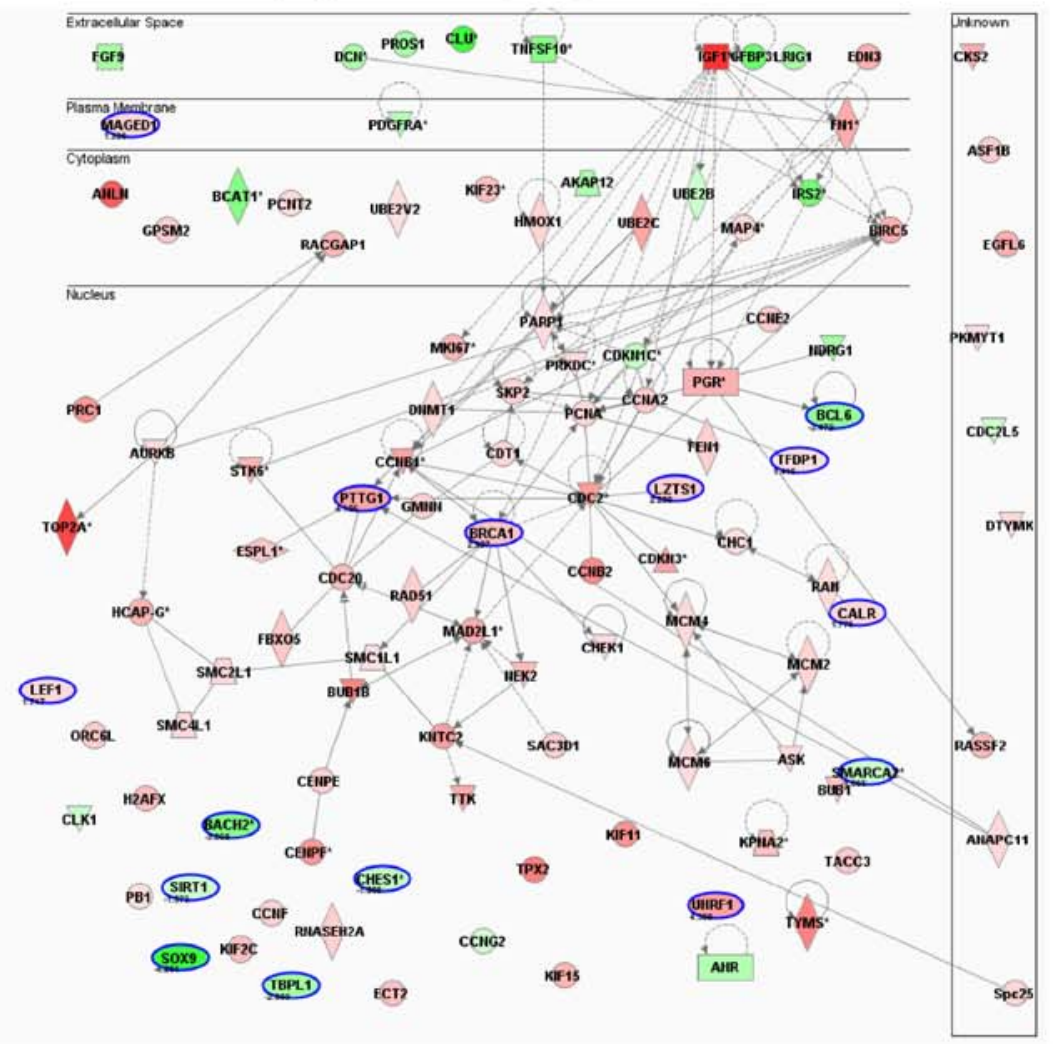

b

Tibolone regulated cell cycle genes

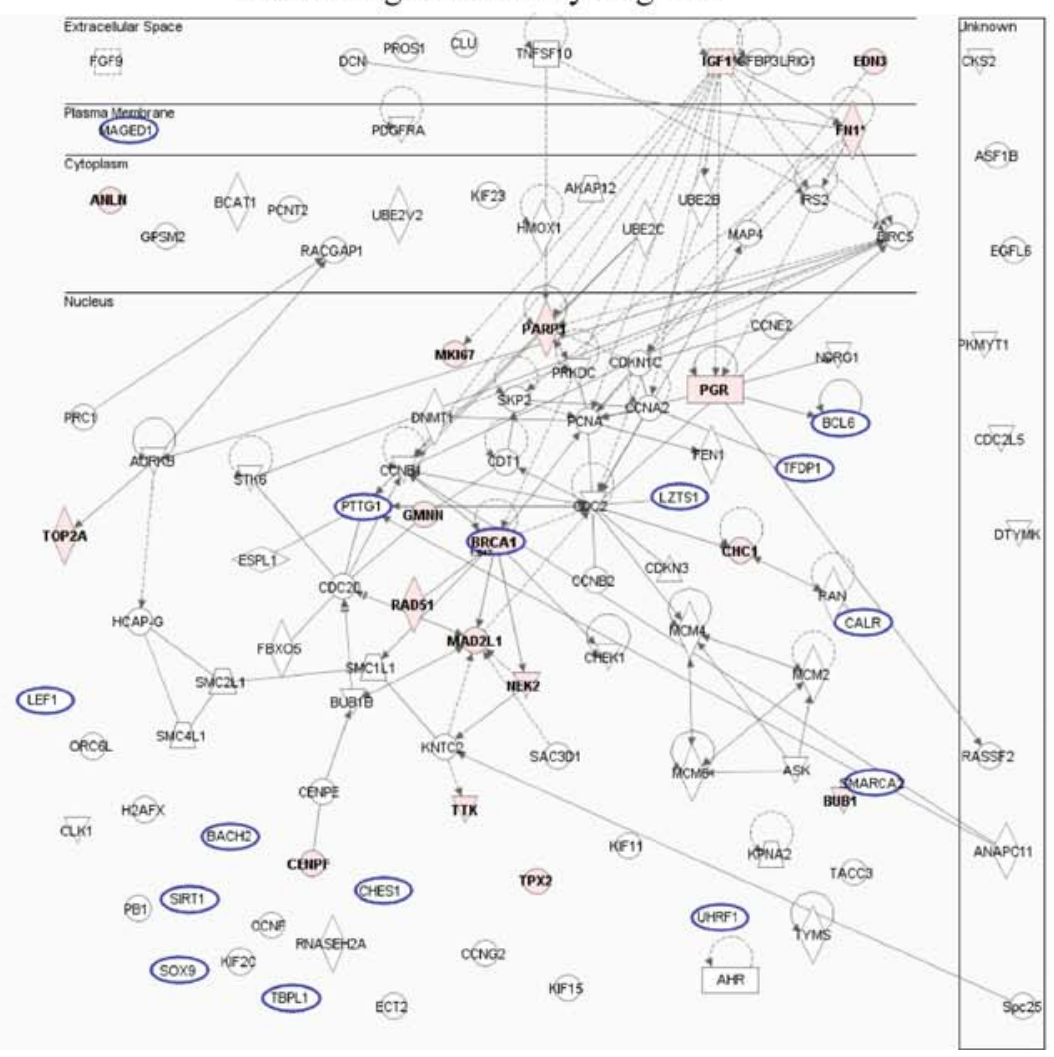


4Fig. 3 A gene expression network of $E_{2^{-}}$and tibolone-regulated genes involved in the regulation of the cell cycle. From top to bottom, genes are acting in extracellular space, plasma membrane, cytoplasm, or nucleus. The colors indicate upregulation (red), downregulation (green) or no regulation (not colored). The intensity of colors indicates the magnitude of regulation (darker means more pronounced). Relations between genes are indicated with gray lines and functions of genes by the shape of the gene name boxes. Rectangle Nuclear receptor, square cytokine, vertical diamond enzyme, horizontal diamond peptidase, triangle kinase, quadrangle transporter, ellipse transcription factor, circle other function

patients, and 174 genes are significantly regulated in endometria from E2 + MPA treated patients. These results indicate that $E_{2}$ treatment has a much higher impact on endometrial RNA expression than the other treatments.

Upon evaluating the overlap in signaling, it was observed that $9 \%$ of $E_{2}$-regulated genes are also regulated by tibolone (72 out of 799) and 5\% are also regulated by E2 + MPA treatment (43 out of 799). The overlap between tibolone and E2 + MPA treatment is about 10\% (17 out of 173) and between tibolone and $\mathrm{E}_{2}$ treatment is $42 \%$ (72 out of 173 ). Most regulated genes, however, are not regulated by more than one treatment. These results indicate that all three treatments result in modulation of expression of, for the majority, uniquely regulated genes (the numbers of uniquely regulated genes are indicated by the italicized numbers in Table 2) and that a subset of tibolone-regulated genes $(42 \%)$ overlaps with a limited fraction $(9 \%)$ of $E_{2}$-regulated genes. The gene list resulting from the SAM analysis is posted at http://www2.eur.nl/fgg/rede/hanifi_moghaddam/.

Functional and biological classification of genes

To gain insight into biological processes affected by the different hormonal treatments (relative to control), genes

Fig. 4 Array data compared to RT-PCR results. The array signal intensities (top) of tested genes were normalized relative to GAPDH. The RT-PCR signals (bottom) were normalized relative to 18 sRNA signals. Solid black bars represent data from control patients, solid white bars represent data from tibolone-treated patients, dashed gray bars represent data from $\mathrm{E}_{2}+$ MPA treated patients, and the solid gray bars represent data from $\mathrm{E}_{2}$-treated patients

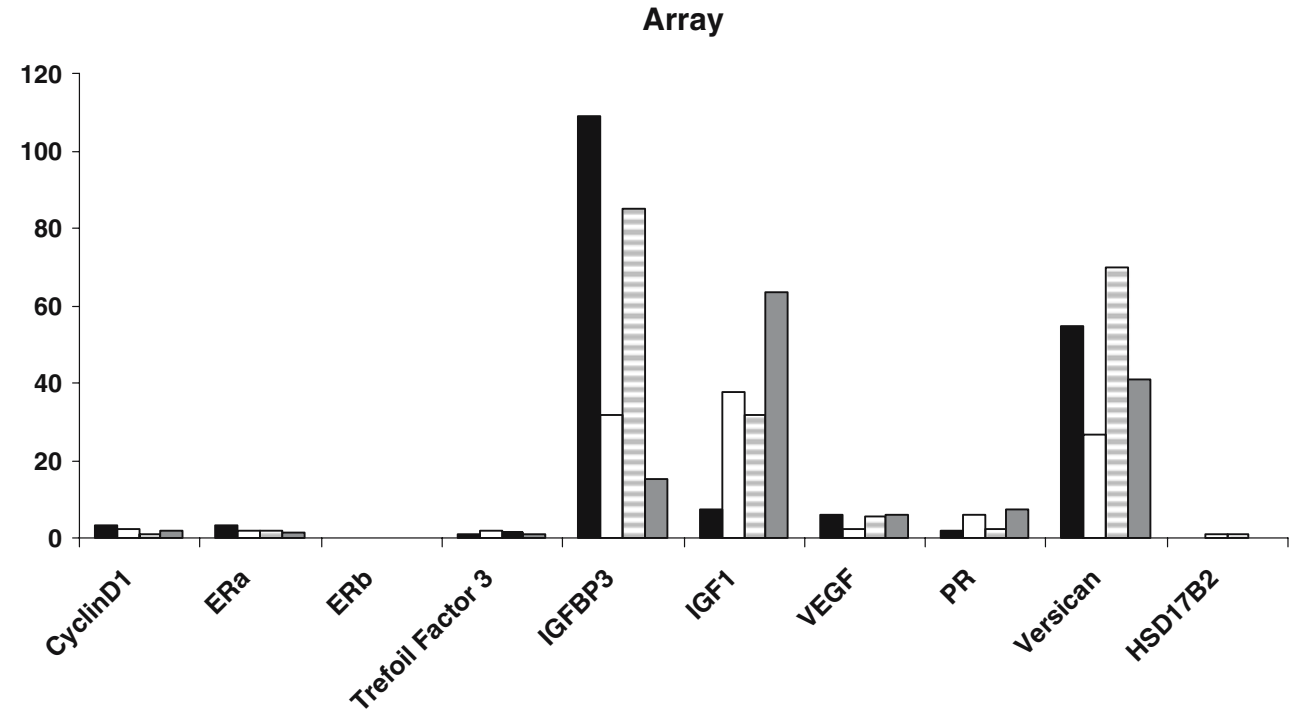

\section{Real Time RT-PCR}

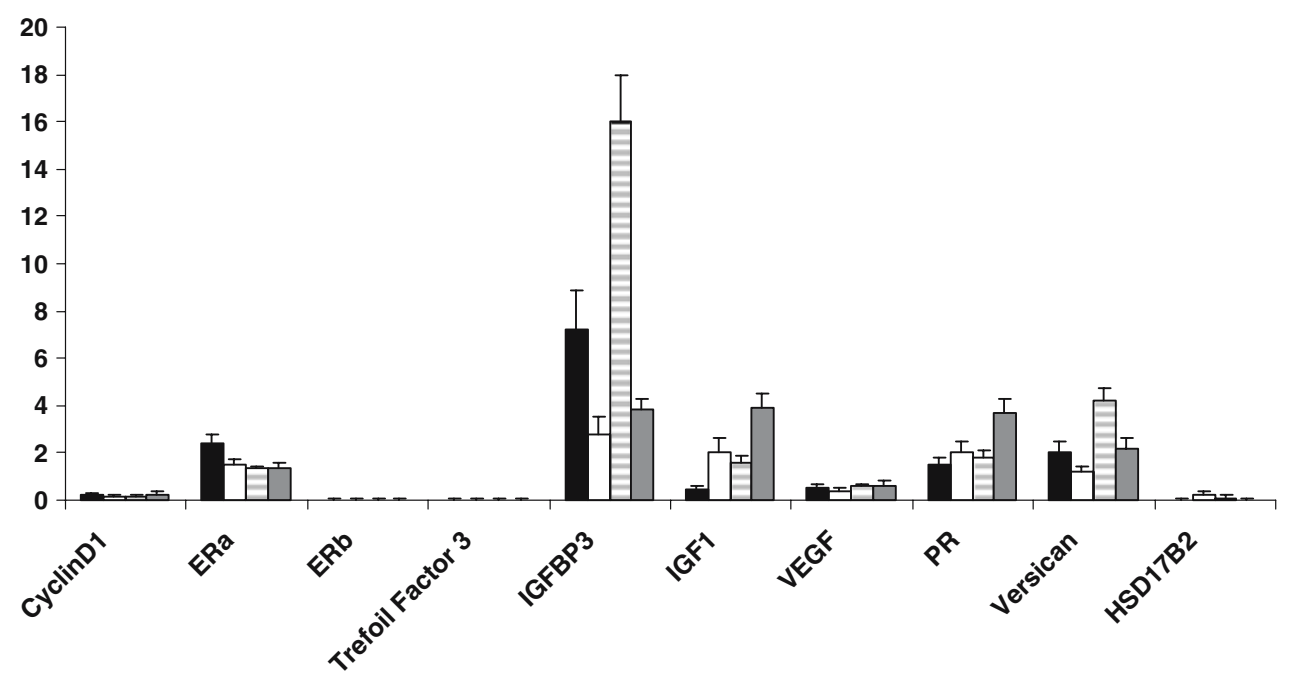


significantly regulated in each treatment group were classified into biological processes (Supplementary Table 3 posted at http://www2.eur.nl/fgg/rede/hanifi_moghaddam/).

The overlap between $\mathrm{E}_{2}$ and tibolone treatment was further evaluated at the level of the cell cycle. In total 112 cell cycle genes were regulated by $\mathrm{E}_{2}$ ( 87 genes upregulated, 25 genes downregulated). The $\mathrm{E}_{2}$ treatment shared only 18 genes with the tibolone treatment (a total of 22 cell cycle genes were regulated by tibolone treatment). In Fig. 3 most of these data were placed in a gene expression network containing information on the gene name, degree of downregulation or upregulation, general function, connectivity to other genes in the network, and general site of action. As indicated by number of regulated genes and the intensities of the colors in Fig. 3, it is clear that $E_{2}$ treatment has a far more pronounced effect on cell cycle genes than tibolone. Survey of the overlapping genes showed that the majority of genes belonged to the families of enzymes and transcription regulators, and out of the 15 transcription regulators that were regulated by $\mathrm{E}_{2}$, only one was also regulated by tibolone treatment (Fig. 3). The gene list used to generate Fig. 3 is posted at http://www2.eur.nl/fgg/rede/ hanifi_moghaddam/.

\section{Verification of array results}

In Fig. 4, the average outcomes of the quantitative PCR analysis on CCND1, TFF3, IGFBP3, IGF1, VEGF, CSPG2, $\mathrm{ER} \alpha, \mathrm{ER} \beta, \mathrm{PRB}$, and HSD17B2 are compared to the average outcomes of the microarray experiments. Although the absolute values cannot be compared, it was observed that the RT-qPCR values resembled the microarray data quite well.

\section{Discussion}

The current study is part of a multidisciplinary effort (histological, biochemical, and molecular) to document shortterm (21 days) effects of tibolone on the human postmenopausal endometrium, in comparison to the effects of short-term $\mathrm{E}_{2}$ and $\mathrm{E} 2+\mathrm{MPA}$ treatments. In this report we show for the first time that the 21-day treatment of postmenopausal patients with $\mathrm{E}_{2}, \mathrm{E} 2+\mathrm{MPA}$, or tibolone results in unique changes in the endometrial gene expression profiles.

Gene expression profiles and regulated processes

Based on the results of cluster and correlation analyses, we showed that the endometrial profiles after tibolone treatment display a weak similarity to the profiles after $E_{2}$ treatment and no similarity to control profiles or profiles after E2 + MPA treatment. Upon analyzing the actual numbers of significantly regulated genes and the overlap in gene regulation between the treatments, this observation could be refined further. Based on the number of regulated genes, it was determined that the impact of $E_{2}$ treatment (799 significantly regulated genes) on endometrial gene expression was much more pronounced than the impact of the E2 + MPA (174 significantly regulated) or tibolone (173 significantly regulated genes) treatments. Furthermore, all treatments resulted in regulation of mostly treatmentspecific genes: $87 \%$ (695 out of 799) of $\mathrm{E}_{2}$-regulated genes were $E_{2}$-specific, $72 \%$ (125 out of 174) of E2 + MPA regulated genes were treatment-specific, and 55\% (95 out of 173) of tibolone regulated genes were tibolone-specific. The overlap in gene regulation between the treatments showed the highest overlap between tibolone- and $E_{2}$ regulated genes. The absolute number of overlapping genes is 72 , which represents $42 \%$ of tibolone regulated genes, but only $9 \%$ of $E_{2}$ regulated genes. This indicates that tibolone treatment results in regulation of a minor subset of $\mathrm{E}_{2}$-regulated genes. It should also be noted in this respect that the fold modulation of these tibolone/ $\mathrm{E}_{2}$-regulated genes is much more pronounced in the endometria of $E_{2^{-}}$ treated women (average fold induction is 2.93) than in the endometria of the tibolone-treated women (average fold induction is 1.97).

Subsequently, the regulated genes were classified into biological processes. Treatment of women with E2 + MPA does not result in the regulation of any, by the Panther database predefined, biological processes. This result was not totally unexpected because cluster and correlation analysis grouped the endometrial profiles after E2 + MPA treatment with the endometrial profiles of untreated women (control). However, upon a gene to gene base review of expression, we could show specific regulation of a number of implantation- and decidualization-related genes: fibrillin [20], hydroxyprostaglandin dehydrogenase [21], glycodelin [22], IGFBP1 [23], LEFTY2 [24], osteopontin [25], granzymeA and B [26], glutathione peroxidase [27] and more. Regulation of these genes is consistent with a profound effect of progesterone on implantation and early pregnancy.

It was also shown that tibolone and $\mathrm{E}_{2}$ treatments result in significant regulation of processes involved in cell cycle regulation. Upon placing the $\mathrm{E}_{2^{-}}$and tibolone-regulated genes involved in cell cycle regulation in one gene expression network, it becomes clear that estrogens regulate the cell cycle far more extensively than tibolone does.

Comparing the molecular data with earlier assessed histological data

Before treatment started, all patients were assessed as postmenopausal with an inactive endometrium. Upon 
measuring endometrial thickness after treatment, effects of the hormonal substitutions were observed [14]. Endometrial thickness increased by $0.5 \mathrm{~mm}$ to $1.0 \mathrm{~mm}( \pm 0.1)$ after tibolone treatment, increased to $1.1 \mathrm{~mm}(( \pm 0.6)$ after E2 + MPA treatment, and increased to $2.6 \mathrm{~mm}\left(( \pm 1.6)\right.$ after $\mathrm{E}_{2}$ treatment. Assessment of proliferation showed an atrophic/ inactive endometrium in the control and E2 + MPA group. Tibolone treatment resulted in a small stimulation, while the endometria of $\mathrm{E}_{2}$-only treated women displayed pronounced stimulation. Using Ki67 staining (a marker for mitotic activity), the increment in proliferation could be assessed. In tibolone-treated endometria in comparison to control, Ki67 showed 3.5-fold more staining in stromal and approximately the same staining in glandular cells. For E2 + MPA treatment, these figures were 6.5 -fold more in stromal and 0.5 -fold less in glandular cell, while for $\mathrm{E}_{2}$ only treatment, again there was pronounced stimulation: 26.5-fold more staining in stromal cells and 6.6-fold more staining in glandular cells (microarrays showed significant upregulation of MKI67 only in $\mathrm{E}_{2}$ treated patients). The overall conclusion of the histological evaluation was that short-term (21 days) tibolone and E2 + MPA treatments resulted in a small stimulation of proliferation of mostly the stromal compartment of the endometrium, and that this stimulation clearly differed from $\mathrm{E}_{2}$ stimulation of the endometrium. Furthermore, it was also clear that the tibolone and E2 + MPA treatments did not display exactly the same effects on the endometria. Upon comparing the histological findings with the currently discussed molecular findings it is clear that both sets of data, for most part, result in very similar conclusions.

Comparing the molecular data with earlier assessed biochemical [15] data

During passage through the liver and gastrointestinal tract, tibolone is converted into three metabolites: two hydroxy metabolites, $3 \alpha-\mathrm{OH}$-tibolone and $3 \beta-\mathrm{OH}$-tibolone (exerting estrogenic effects), and the $\Delta^{4}$-isomer (exerting progestagenic and androgenic effects) [7, 9]. Hormone measurements showed that the metabolite patterns of tibolone in myometrium (due to tissue shortage the myometrial layer just beneath the endometrium was used for measuring hormones) are different from those in serum [15]. Whereas low levels of bioactive metabolites (unconjugated) are found in serum, $\Delta^{4}$-isomer levels are high in the myometrium of most women. This indicates that biochemically in serum, tibolone displays weak estrogenic activity, while in the uterus, tibolone progestagenic activities are more pronounced. This biochemical finding is of interest because it may help explain why the short-term effects of tibolone on gene expression are pronouncedly different from the effects of $\mathrm{E}_{2}$ alone and also different from the effects of E2 + MPA.
In summary, by making use of state of the art bioinformatic tools, a detailed analysis could be conducted, which refined our initial cluster analysis and showed that tibolone signaling displays a very limited overlap with estrogen signaling, that tibolone displays no overlap with E2 + MPA signaling, and that tibolone treatment results mostly in regulation of a treatment-specific set of genes in the endometrium. Furthermore, the current data are in agreement with the earlier published histological assessment of the same endometrial tissues and seem to be supported by the biochemical assessment of the metabolites of tibolone.

Acknowledgement This work was sponsored by N.V. Organon, Oss, The Netherlands.

\section{References}

1. Pickar JH, Yeh IT, Wheeler JE, Cunnane MF, Speroff L (2003) Endometrial effects of lower doses of conjugated equine estrogens and medroxyprogesterone acetate: two-year substudy results. Fertil Steril 80:1234-1240

2. Pickar JH, Yeh I, Wheeler JE, Cunnane MF, Speroff L (2001) Endometrial effects of lower doses of conjugated equine estrogens and medroxyprogesterone acetate. Fertil Steril 76:25-31

3. Anderson GL, Limacher M, Assaf AR, Bassford T, Beresford SA, Black H, Bonds D, Brunner R, Brzyski R, Caan B, Chlebowski R, Curb D, Gass M, Hays J, Heiss G, Hendrix S, Howard BV, Hsia J, Hubbell A, Jackson R, Johnson KC, Judd H, Kotchen JM, Kuller $\mathrm{L}$, LaCroix AZ, Lane $\mathrm{D}$, Langer $\mathrm{RD}$, Lasser $\mathrm{N}$, Lewis $\mathrm{CE}$, Manson J, Margolis K, Ockene J, O'Sullivan MJ, Phillips L, Prentice RL, Ritenbaugh C, Robbins J, Rossouw JE, Sarto G, Stefanick ML, Van Horn L, Wactawski-Wende J, Wallace R, Wassertheil-Smoller S; Women's Health Initiative Steering Committee (2004) Effects of conjugated equine estrogen in postmenopausal women with hysterectomy: the Women's Health Initiative randomized controlled trial. JAMA 291:1701-1712

4. Beral V, Million Women Study Collaborators (2003) Breast cancer and hormone-replacement therapy in the Million Women Study. Lancet 362:419-427

5. Kloosterboer HJ (2004) Tissue-selectivity: the mechanism of action of tibolone. Maturitas 48(Suppl 1):S30-S40

6. Kloosterboer HJ (2004) Tissue-selective effects of tibolone on the breast. Maturitas 49:S5-S15

7. Kloosterboer HJ (2001) Tibolone: a steroid with a tissue-specific mode of action. J Steroid Biochem Mol Biol 76:231-238

8. Timmer CJ, Verheul HA, Doorstam DP (2002) Pharmacokinetics of tibolone in early and late postmenopausal women. Br J Clin Pharmacol 54:101-106

9. de Gooyer ME, Deckers GH, Schoonen WG, Verheul HA, Kloosterboer HJ (2003) Receptor profiling and endocrine interactions of tibolone. Steroids 68:21-30

10. Tang B, Markiewicz L, Kloosterboer HJ, Gurpide E (1993) Human endometrial 3 beta-hydroxysteroid dehydrogenase/isomerase can locally reduce intrinsic estrogenic/progestagenic activity ratios of a steroidal drug (Org OD 14). J Steroid Biochem Mol Biol 45:345-351

11. Schatz F, Kuczynski E, Kloosterboer HJ, Buchwalder L, Tang C, Krikun G, Lockwood CJ (2005) Tibolone and its metabolites enhance tissue factor and PAI-1 expression in human endometrial 
stromal cells: evidence of progestogenic effects. Steroids 70:840845

12. Hanifi-Moghaddam P, Gielen SC, Kloosterboer HJ, De Gooyer ME, Sijbers AM, van Gool AJ, Smid M, Moorhouse M, van Wijk FH, Burger CW, Blok LJ (2005) Molecular portrait of the progestagenic and estrogenic actions of tibolone: behavior of cellular networks in response to tibolone. J Clin Endocrinol Metab 90:973-983

13. Falany JL, Macrina N, Falany CN (2004) Sulfation of tibolone and tibolone metabolites by expressed human cytosolic sulfotransferases. J Steroid Biochem Mol Biol 88:383-391

14. Klaassens AH, van Wijk FH, Hanifi-Moghaddam P, Sijmons B, Ewing PC, Ten Kate-Booij MJ, Kooi GS, Kloosterboer HJ, Blok LJ, Burger CW (2006) Histological and immunohistochemical evaluation of postmenopausal endometrium after 3 weeks of treatment with tibolone, estrogen only, or estrogen plus progestagen. Fertil Steril 86:352-361

15. Verheul HAM, Blok LJ, Burger CW, Hanifi-Moghaddam P, Kloosterboer HJ (2006) Levels of unconjugated and sulfated metabolites of tibolone and estradiol in serum, myometrium and vagina of postmenopausal women treated for 21 days with tibolone, estradiol or estradiol plus medroxyprogesterone acetate. J Soc Gynecol Investig (in press)

16. Bolstad BM, Irizarry RA, Astrand M, Speed TP (2003) A comparison of normalization methods for high density oligonucleotide array data based on variance and bias. Bioinformatics 19:185-193

17. Tusher VG, Tibshirani R, Chu G (2001) Significance analysis of microarrays applied to the ionizing radiation response. Proc Natl Acad Sci USA 98:5116-5121

18. Deng L, Shipley GL, Loose-Mitchell DS, Stancel GM, Broaddus R, Pickar JH, Davies PJ (2003) Coordinate regulation of the production and signaling of retinoic acid by estrogen in the human endometrium. J Clin Endocrinol Metab 88:2157-2163

19. Odmark IS, Carlstrom K, Jonsson B, Jonasson AF (2006) Conjugated estrogen/progestagen versus tibolone hormone re- placement therapy in postmenopausal women: effects on carbohydrate metabolism and serum sex hormone-binding globulin. Maturitas 53:89-96

20. Fleming S, Bell SC (1997) Localization of fibrillin-1 in human endometrium and decidua during the menstrual cycle and pregnancy. Hum Reprod 12:2051-2056

21. Cameron ST, Critchley HO, Buckley CH, Kelly RW, Baird DT (1997) Effect of two antiprogestins (mifepristone and onapristone) on endometrial factors of potential importance for implantation. Fertil Steril 67:1046-1053

22. Mueller MD, Vigne JL, Vaisse C, Taylor RN (2000) Glycodelin: a pane in the implantation window. Semin Reprod Med 18:289298

23. Kim JJ, Fazleabas AT (2004) Uterine receptivity and implantation: the regulation and action of insulin-like growth factor binding protein-1 (IGFBP-1), HOXA10 and forkhead transcription factor1 (FOXO-1) in the baboon endometrium. Reprod Biol Endocrinol $2: 34$

24. Tang M, Mikhailik A, Pauli I, Giudice LC, Fazelabas AT, Tulac S, Carson DD, Kaufman DG, Barbier C, Creemers JW, Tabibzadeh S (2005) Decidual differentiation of stromal cells promotes proprotein convertase 5/6 expression and lefty processing. Endocrinology 146:5313-5320

25. von Wolff M, Bohlmann MK, Fiedler C, Ursel S, Strowitzki T (2004) Osteopontin is up-regulated in human decidual stromal cells. Fertil Steril 81(Suppl 1):741-748

26. Hayakawa S, Nagai N, Kanaeda T, Karasaki-Suzuki M, Ishii M, Chishima F, Satoh K (1999) Interleukin-12 augments cytolytic activity of peripheral and decidual lymphocytes against choriocarcinoma cell lines and primary culture human placental trophoblasts. Am J Reprod Immunol 41:320-329

27. Riesewijk A, Martin J, van Os R, Horcajadas JA, Polman J, Pellicer A, Mosselman S, Simon C (2003) Gene expression profiling of human endometrial receptivity on days $\mathrm{LH}+2$ versus $\mathrm{LH}+7$ by microarray technology. Mol Hum Reprod 9: 253-264 